\title{
A novel 5-Plex qPCR-HRM assay detecting human diarrheal parasites
}

\author{
Aline Lamien-Meda* ${ }^{*}$, Renate Schneider, Julia Walochnik, Herbert Auer, Ursula Wiedermann and David Leitsch
}

\begin{abstract}
Background: Intestinal parasitic diseases occur worldwide, and their diagnosis poses considerable challenges. Cryptosporidium spp., Entamoeba histolytica, Giardia intestinalis, (and, arguably, Dientamoeba fragilis and Blastocystis spp.) are among the most important and common parasitic protozoans causing diarrhea. Several multiplex real-time PCR assays have been developed for the synchronous detection of these parasites. However, most assays include the use of hydrolysis probes, increasing the cost of stool examination. In this study, we designed and evaluated a real-time PCR protocol, based on high-resolution melting (HRM) curve analysis, to simultaneously detect and differentiate five gastrointestinal parasites.

Results: Using a blinded panel of 143 clinical samples with laboratory diagnostic data to evaluate the method, we obtained a $95.8 \%$ concordance with conventional methods. Moreover, $4.2 \%$ of the samples were positive for D. fragilis and 2.8\% additional Cryptosporidium infections were found with our multiplex assay. Our method is sensitive and specific for the selected parasites with the additional possibility of being run in single-plex as a backup control for mixed infections.

Conclusions: The assay is a convenient and cost-effective method that could contribute to a quicker and accurate diagnosis as well as to more targeted therapies of parasite-derived diarrhea. Finally, this new multiplex PCR assay could also be instrumental in epidemiology studies on these parasites.
\end{abstract}

Keywords: Diarrhea, qPCR-HRM, Dientamoeba, Entamoeba, Cryptosporidium, Blastocystis, Giardia

\section{Background}

Human intestinal protozoan infections can lead to significant morbidity and mortality when not diagnosed timely and treated appropriately. Such infections are typically more associated with low-income countries. But with worldwide traveling and migration increasing in importance, there is also a continuous increase in the number of cases in industrialized countries. The diagnosis of intestinal protozoan diseases has remained a challenge, especially in low-endemicity countries where practitioners and health institutions are less familiar with the respective causative agents [1].

*Correspondence: aline.lamienmeda@meduniwien.ac.at Institute for Specific Prophylaxis and Tropical Medicine, Center for Pathophysiology, Infectiology and Immunology, Medical University of Vienna, Kinderspitalgasse 15, 1090 Vienna, Austria
Cryptosporidium spp., Entamoeba histolytica, and Giardia intestinalis are the most important parasitic protozoans causing diarrhea [2, 3]. Giardiasis and cryptosporidiosis are major causes of moderate to severe diarrhea in both, developing and developed countries, leading to considerable mortality worldwide $[2,3]$. Amoebiasis is the third most frequent cause of death from protozoan parasitic diseases, with high prevalence in developing countries $[4,5]$.

Blastocystis spp. and Dientamoeba fragilis are very common protozoan commensals whose pathogenic potential is still under evaluation [6]. Human infection rates with Blastocystis spp. can range between 1-60\% and can even go up to $100 \%$, depending on the geographic distribution and also the economic status of a given country $[10,11]$. D. fragilis is a trichomonad parasite [4] 
of the human gastrointestinal tract and has, since 1919, been repeatedly reported in symptomatic patients [6]. The incidence of $D$. fragilis is equal to or even exceeds that of G. intestinalis [7].

As several intestinal protozoans can cause diarrhea, it is important to use diagnostic methods capable of easily and accurately identifying the infectious agents. A multiplex PCR approach can address challenges as presented by microscopy and immunofluorescence assays, such as the dependence on well-trained microscopists, and time or sensitivity issues. Despite the limitations, microscopy is still considered as the reference standard method for routine diagnostics due to its low cost and practicability in endemic countries. The main benefits of a multiplex PCR include cost-effectiveness, time-saving, and higher throughput. Nevertheless, the design and evaluation of a multiplex assay require an investment in terms of time and costs for optimization and validation.

In this study, we established a cost-effective and practical tandem multiplex-high resolution melting analysis for simultaneous detection and quantification of five intestinal protozoa: Cryptosporidium spp., E. histolytica, Giardia intestinalis (assemblages A and B), Blastocystis spp., and D. fragilis.

\section{Results}

\section{Assay design and optimization}

The individual primer sets used for the multiplex PCR were designed based on sequences obtained from the literature for the generation of long fragments (Table 1). These fragments were submitted to BLAST and used for the design of shorter fragments for the multiplex PCR. Each short fragment was selected according to its specificity, fidelity, and predicted melting temperature. The short fragment primers were designed using the online Primer 3 software (http://bioinfo.ut.ee/primer3-0.4.0/) [8]. Table 1 is presenting details of all primers from the long and the short fragments from the respective target gene for each parasite species.

The 5-plex qPCR is specifically differentiating Blastocystis spp. (Blastocystis), Cryptosporidium spp. (Cryptosporidium), D. fragilis (Dientamoeba), E. histolytica (Entamoeba), G. intestinalis assemblage A (Giardia assemblage A), and G. intestinalis assemblage B (Giardia assemblage $B$ ) through the melting temperature of their selected and amplified fragment (Fig. 1). We also used individual primer sets in a single-plex run for the identification of each parasite species.

Interestingly the plasmids were presenting the same melting peak in both multiplex and single-plex runs. The melting temperature $(\mathrm{Tm})$ values were $71.50 \pm 0.00{ }^{\circ} \mathrm{C}$ (Dientamoeba), $\quad 75.20 \pm 0.25 \quad{ }^{\circ} \mathrm{C} \quad$ (Entamoeba), $78.23 \pm 0.25 \quad{ }^{\circ} \mathrm{C} \quad$ (Cryptosporidium), $\quad 79.84 \pm 0.23 \quad{ }^{\circ} \mathrm{C}$
(Blastocystis), $81.51 \pm 0.08$ (Giardia assemblage B) and $83.50 \pm 0.00{ }^{\circ} \mathrm{C}$ (Giardia assemblage A) (Fig. 2).

\section{Efficiency and limit of detection (LOD) of the assay}

The sensitivity of the multiplex was tested by amplifying individually tenfold serial dilutions of plasmids containing copies ( $\leq 10^{7}$ copies) of the respective targeted gene fragment each. The multiplex assay detected target gene numbers as low as 100 copies/ $\mu \mathrm{l}$ (for Giardia assemblage B) or even ten copies/ $\mu \mathrm{l}$ (for Blastocystis, Cryptosporidium, Dientamoeba, Entamoeba, and Giardia assemblage $A)$. The ranges of efficiency, slope, and $\mathrm{R}^{2}$ were 95.77 to $103.11 \%,-3.2497$ to -3.4277 and 0.9942 to 0.9998 respectively (Table 2 ).

Further dilutions were prepared (between 200 and 1 copy $/ \mu \mathrm{l})$ to determine the LOD for each parasite. The calculated LODs at 95\% confidence for the multiplex method using probit analysis varied from 8.78 (6.0615.99) copies/ $\mu$ l for Cryptosporidium to 30.08 (18.0269.13) copies/ $\mu \mathrm{l}$ for Entamoeba (Table 2).

The Welch's unequal variances $t$ test showed that all Tm's were significantly different from each other as in the predicted melting, with $\Delta \mathrm{Tm}$ (between the closest Tms) value of $3.7^{\circ} \mathrm{C}$ (Dientamoeba/Entamoeba), $3.03{ }^{\circ} \mathrm{C}$ (Ent amoeba/Cryptosporidium), $1.61{ }^{\circ} \mathrm{C}$ (Cryptosporidium/Bl astocystis), $2.67{ }^{\circ} \mathrm{C}$ between (Blastocystis/Giardia assemblage B), and $1.99{ }^{\circ} \mathrm{C}$ (Giardia assemblage B/Giardia assemblage A) (Table 2).

\section{Sample genotyping and multiplex specificity}

The discriminatory power of the multiplex PCR was tested using 143 DNA isolates from our routine diagnostic laboratory archive. We tested blinded samples using the multiplex method, and the results were compared to previous diagnostic results obtained through microscopy, immunoassays, and/or FRET-based qPCR. The DNA of Entamoeba coli, Entamoeba hartmanni, Iodamoeba buetschlii and 13 other organisms listed in the methods section was included in the tests to confirm the specificity of the method. The negative samples included parasite-free human stool samples and Entamoeba dispar samples. Figure 3 juxtaposes the multiplex PCR results with the original results from the routine diagnostic unit. Up to $95.8 \%$ (137) of our results were matching with the routine diagnostics. The 5-Plex method was able to detect four (2.8\%) more Cryptosporidium infections, which were confirmed by DNA sequencing and also with Verweij et al. primers (Table 1) [9], to be C. parvum, and six (4.2\%) samples were found to be positive for $D$. fragilis. Details on non-concordant samples with $(8.4 \%)$ and without (4.2\%) Dientamoeba data are presented in Table 3. 
Table 1 List of primers for amplification of the long original and the short new fragments, with fragment sizes and target genes for each parasite species

\begin{tabular}{|c|c|c|c|}
\hline Species \& Gene & Primers & PCR product & Reference \\
\hline \multirow[t]{3}{*}{ Dientamoeba fragilis } & Long fragment & $886 \mathrm{bp}$ & [9] \\
\hline & Dienta F: 5'-TAT CGG AGG TGG TAA TGA CC-3' & & \\
\hline & Dienta R: 5'-CAT CTT CCT CCT GCTTAG ACG-3' & & \\
\hline \multirow[t]{3}{*}{ SSU rRNA } & Short fragment & $114 \mathrm{bp}$ & Our study \\
\hline & Dienta F2: 5'-CAA ATC AGA ACG CTT AAA GTA ATT TTC-3' & & \\
\hline & Dienta R2: 5'-CCC CGA TTA TTC TCT TTG ATA TT-3' & & \\
\hline \multirow[t]{3}{*}{ Entamoeba histolytica } & Long fragment & $167 \mathrm{bp}$ & {$[20]$} \\
\hline & EntaF: 5'-ATG CAC GAG AGC GAA AGC AT-3' & & \\
\hline & EhR: 5'-GAT CTA GAA ACA ATG CTT CTC T-3' & & \\
\hline \multirow[t]{3}{*}{ SSU rRNA } & Short fragment & $96 \mathrm{bp}$ & Our study \\
\hline & EntaF2: 5'-CGA TCA GAT ACC GTC GTA GTC C-3' & & \\
\hline & EhR: 5'-GAT CTA GAA ACA ATG CTT CTC T-3' & & \\
\hline \multirow[t]{3}{*}{ Cryptosporidium spp. } & Long fragment & $\sim 161 \mathrm{bp}$ & {$[21]$} \\
\hline & Crypto F: 5'-AGT GAC AAG AAA TAA CAA TAC AGG -3' & & \\
\hline & Crypto R: 5'-CCT GCTTTA AGC ACT CTA ATTTTC-3' & & \\
\hline \multirow[t]{6}{*}{ SSU rRNA } & Short fragment & $\sim 88 \mathrm{bp}$ & Our study \\
\hline & Crypto_Frag_F: 5'-GTG ACA TAT CATTCA AGT TTC TGA CC-3' & & \\
\hline & Crypto_Frag_R: 5'-TAA TTC CCC GTT ACC CGT CA-3' & & \\
\hline & Confirmation qPCR-HRM & $138 \mathrm{bp}$ & {$[9]$} \\
\hline & CrF 5'-CGC TTC TCT AGC CTTTCA TGA-3 & & \\
\hline & CrR 5'-CTT CAC GTG TGTTTG CCA AT-3 & & \\
\hline \multirow[t]{3}{*}{ Blastocystis spp. } & Long fragment & $320-342 \mathrm{bp}$ & {$[22]$} \\
\hline & BI_18rRNA F: 5'-AGT AGT CAT ACG CTC GTC TCA AA-3' & & \\
\hline & BI_18rRNA R: 5'-TCTTCG TTA CCC GTT ACT GC-3' & & \\
\hline \multirow[t]{3}{*}{ SSU rRNA } & Short fragment & 82 bp & Our study \\
\hline & BI_18rRNA_F1: 5'-GCA GTA ACG GGT AAC GAA GAA-3' & & \\
\hline & BI_18rRNA_R1: 5'-TGC TGC CTT CCTTGG ATG T-3' & & \\
\hline \multirow[t]{3}{*}{ Giardia intestinalis } & Long fragment & $\sim 432 \mathrm{bp}$ & {$[23]$} \\
\hline & GDHiF: 5'-CAG TAC AAC TCY GCT CTC GG-3 & & \\
\hline & GDHiR: 5'-GTT RTC CTT GCA CAT CTC C-3' & & \\
\hline \multirow[t]{3}{*}{ gdh } & Short fragment & $\sim 133 \mathrm{bp}$ & Our study \\
\hline & Giar-GDH-F3: 5'-GGC AAG AAC RTC AAG TGG-3' & & \\
\hline & Giar-GDH-R2: 5'-TTG TCC TTG CAC ATC TCC TC-3' & & \\
\hline
\end{tabular}

The selected primer sets were highly specific for the target species, and each parasite species was well differentiated by the individual primer set and also by the 5-plex primers mix. The non-pathogenic amoeba species E. dispar Entamoeba coli, Entamoeba hartmanni, Iodamoeba buetschlii and the 13 organisms listed in the section Materials and methods tested negative with our designed primer sets.

The following cases of double and triple infections were identified within the studied samples: Dientamoeba/Blastocystis $(\mathrm{n}=5)$, Dientamoeba/Giardia $(\mathrm{n}=1)$, Entamoeba/Blastocystis $(\mathrm{n}=4), \quad$ Blastocystis/Giardia $\quad(\mathrm{n}=12), \quad$ and
Blastocystis/Entamoeba/Giardia $(\mathrm{n}=1)$ Fig. 4 . Each mix infection was confirmed by the relative single-plex runs using the target plasmids as reference.

\section{Discussion}

PCR and real-time PCR (qPCR) are nowadays well-established in routine diagnostics for fecal parasites. As compared to microscopy, PCR has the advantage to be fast, more sensitive, easy to perform, and not to require a long period of training, as commonly necessary for a good microscopist. PCR also has the advantage of detecting and quantifying multiple pathogens within one reaction. 

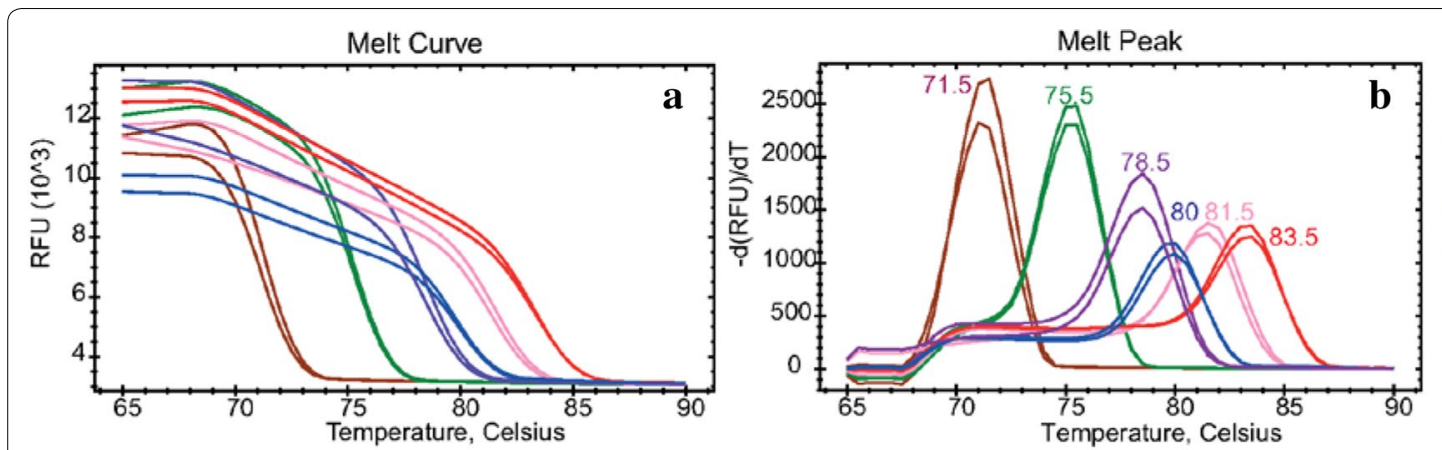

Dientamoeba

Entamoeba

Cryptosporidium

Blastocystis

Giardia A

Giardia B
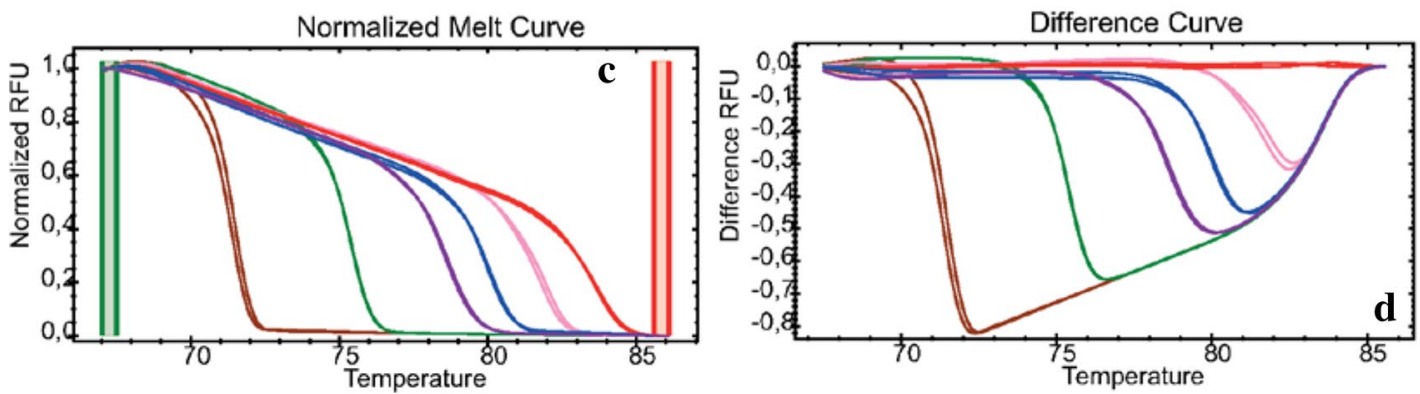

Fig. 1 Melting curves $\mathbf{a}$, melting peaks $\mathbf{b}$, normalized melting peaks $\mathbf{c}$, and differential curves $\mathbf{d}$ of reference plasmids of Blastocystis spp., Cryptosporidium spp., D. fragilis, E. histolytica, G. intestinalis assemblage B and G. intestinalis assemblage A. The following melting temperatures were observed: $71.5^{\circ} \mathrm{C}$ for D. fragilis (brown), $75.5^{\circ} \mathrm{C}$ for E. histolytica (green), $78.5^{\circ} \mathrm{C}$ for Cryptosporidium spp. (lila), $80^{\circ} \mathrm{C}$ for Blastocystis spp. (blue), $81.5^{\circ} \mathrm{C}$ for $\mathrm{G}$. intestinalis assemblage B (light red), and $83.5^{\circ} \mathrm{C}$ for $\mathrm{G}$. intestinalis assemblage A (red). The distance between the melting curves is well specified in the difference curve graph $\mathbf{d}$

Although PCR and qPCR are popular in-house testing methods for parasitological stool diagnostics, they are, in most cases, only used as a complementary method to microscopy and/or immunofluorescent analysis $[9,10]$.

In this study, we developed a multiplex real-time PCR (5-plex qPCR) followed by high resolution melting (HRM) curve analysis targeting specifically protists associated with diarrhea in humans. The five targeted protozoa were Blastocystis spp., Cryptosporidium spp., Dientamoeba fragilis, Entamoeba histolytica, and Giardia intestinalis.

To our knowledge, this method is the first non-probebased multiplex targeting up to 5 human diarrheal parasites with well-separated melting temperatures $\left(\Delta \mathrm{Tm}>1.5^{\circ} \mathrm{C}\right)$. Lalonde and Gajadhar (2011) previously developed PCR melting curve analysis method targeting 8 coccidian oocysts (Cyclosopora cayetanensis, C. parvum, Cryptosporidium muris, Toxoplasma gondii, Eimeria bovis, Eimeria acervulina, Isospora suis, and Sarcocystis cruzi), with eight Tm's ranging between 81.66 and $87.19{ }^{\circ} \mathrm{C}$. In this case, a sample with multiple parasites would be difficult to screen using the melting peaks. Our 5-Plex, however, could be used for the screening of six different types of mixed infection samples since our melting peaks are well separated (Fig. 4). Presently, our assay is the only 5-Plex assay currently available as other multiplex tests for diarrheal parasites target either two [11], three $[1,9,12,13]$, or maximal four $[14,15]$ parasites.

Our novel approach harnesses well-differentiated melting temperatures $\left(\Delta \mathrm{Tm} \geq 1.6^{\circ} \mathrm{C}\right.$, Fig. 1$)$, which allows the simultaneous detection and identification of all five parasites including the assemblages A and B of Giardia. The fact that our multiplex method is detecting more Cryptosporidium species (four) than established standard protocols suggests that our assay may be more sensitive as compared to the standard diagnostic qPCR method for C. parvum. Our method identified the same number of Blastocystis-positive samples as compared to established diagnostic methods used at our institute. However, four Blastocystis samples, one mixed Blastocytis + Giardia sample, and one Giardia sample were also positive for $D$. fragilis, highlighting the great advantage of our multiplex method in detecting co-infections (Table 3 ). The ability to simultaneously detect the five relevant parasites in a single reaction could drastically reduce the costs of parasitological stool diagnostics. Indeed, our assay is inexpensive (approximatively 6.65 USD per sample including DNA extraction) as compared to other methods (12 USD for 4 targets, Stark et al. [15]) and can be performed within $1 \mathrm{~h} 25 \mathrm{~min}$. The method is also quantitative and sensitive, 


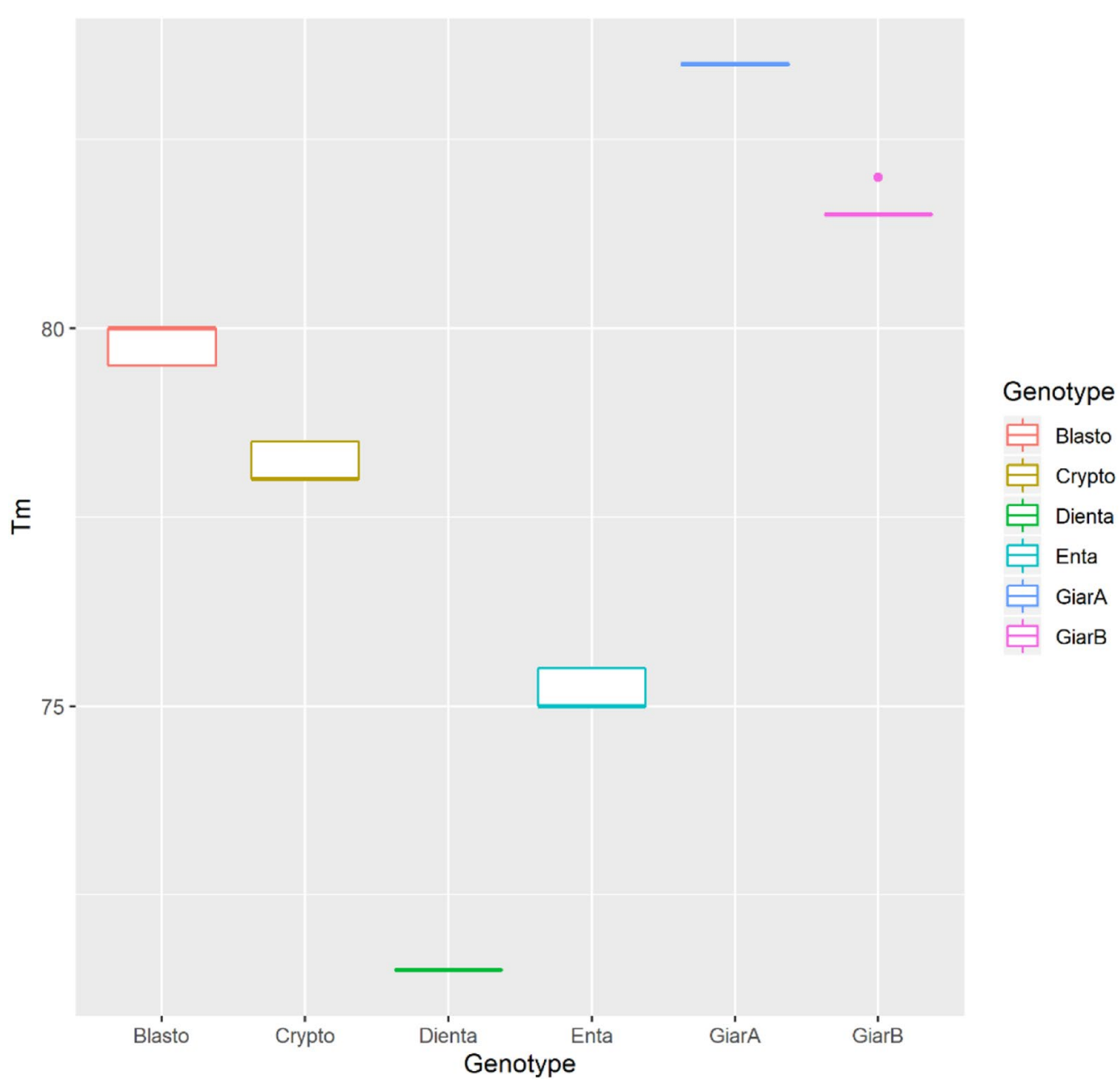

Fig. 2 Boxplots of the melting temperatures (Tm) of Blastocystis spp. (red), Cryptosporidium spp. (brown), D. fragilis (green), E. histolytica (turquoise), Giardia intestinalis assemblage B (pink) and Giardia intestinalis assemblage A (blue). The box indicates the likely range of melting temperature variation. The melting temperature ranges were $71.5-71.5^{\circ} \mathrm{C}$ for Dientamoeba, $75-75.5^{\circ} \mathrm{C}$ for Entamoeba, $78-78.5^{\circ} \mathrm{C}$ for $C r y p t o s p o r i d i u m, 79.5-80^{\circ} \mathrm{C}$ for Blastocystis, $81.5-82^{\circ} \mathrm{C}$ for Giardia assemblage B, and $83-83.5^{\circ} \mathrm{C}$ for Giardia assemblage A. The melting temperature difference $(\Delta \mathrm{Tm})$ between the closest melting curves were $3.7{ }^{\circ} \mathrm{C}$ (Dientamoeba/Entamoeba), $3.03^{\circ} \mathrm{C}$ (Entamoeba/Cryptosporidium), $1.61{ }^{\circ} \mathrm{C}$ (Cryptosporidium/Blastocystis), $1.67^{\circ} \mathrm{C}$ (Blastocystis/Giardia assemblage B) and $2^{\circ} \mathrm{C}$ (Giardia assemblage B/A)

Table 2 Multiplex melting temperatures (Tm), specificities and limits of detection (LOD)

\begin{tabular}{llcccc}
\hline Species & $\operatorname{Tm}\left(\mathbf{T m}\right.$ range) $\left({ }^{\circ} \mathbf{C}\right)$ & Efficiency (\%) & Slope & $\mathbf{R}^{\mathbf{2}}$ & LOD (Min-Max) copy/ $\mathbf{\mu l}$ \\
\hline Dientamoeba fragilis & $71.50 \pm 0.00(71.5-71.5)$ & 98.97 & -3.3468 & 0.9975 & $11.26(7.03-25.15)$ \\
Entamoeba histolytica & $75.20 \pm 0.25(75.0-75.5)$ & 97.05 & -3.3948 & 0.9998 & $30.08(18.02-69.13)$ \\
Cryoptosporidium spp. & $78.23 \pm 0.25(78.0-78.5)$ & 103.11 & -3.2497 & 0.9988 & $8.78(6.06-15.99)$ \\
Blastocystis spp. & $79.84 \pm 0.23(79.5-80.0)$ & 97.60 & -3.3808 & 0.9974 & $10.95(7.08-22.66)$ \\
Giardia intestinalis assemblage B & $81.51 \pm 0.08(81.5-82.0)$ & 95.77 & -3.4277 & 0.9969 & $20.92(15.60-37.15)$ \\
Giardia intestinalis assemblage A & $83.50 \pm 0.00(83.5-83.5)$ & 96.76 & -3.4022 & 0.9942 & $20.00(15.24-33.62)$ \\
\hline
\end{tabular}

with Cryptosporidium presenting the highest sensitivity $(8.78 \mathrm{copy} / \mu \mathrm{l})$ and $E$. histolytica presenting the lowest sensitivity (30.08 copy/ $\mu \mathrm{l})$. We used EvaGreen which seems to be more suitable for multiplex PCR applications as compared to other dyes that are either expensive or may inhibit PCR $[11,16-18]$. 


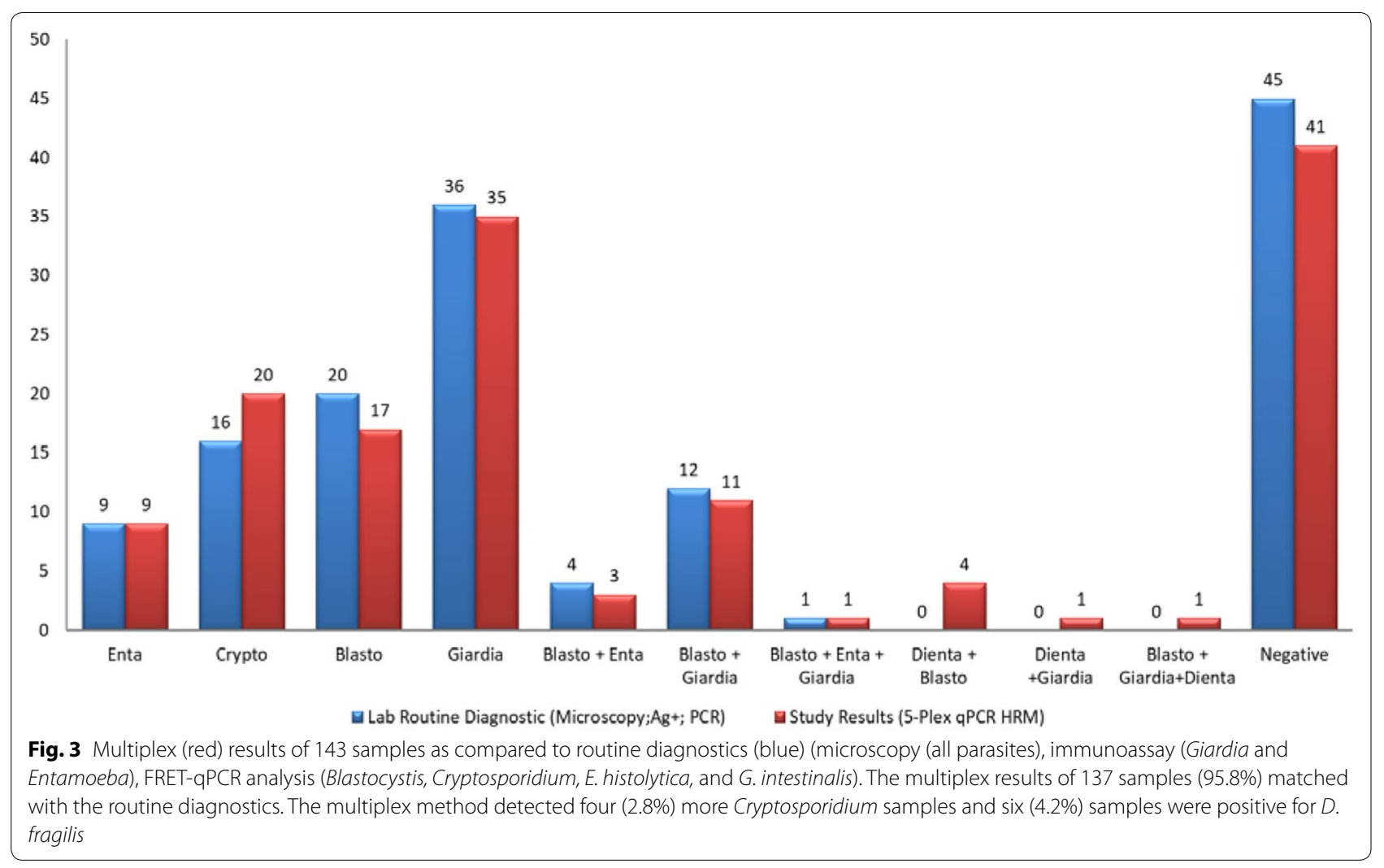

Table 3 List of non-concordant from the 143 samples with their results in both routine diagnostics and the developed 5-Plex-PCR

\begin{tabular}{llll}
\hline Samples & Lab routine diagnostic $\mathbf{a}^{\mathbf{2}}$ & 5-Plex-PCR & Percentage (\%) \\
\hline 20 & Blastocystis & Blastocystis + Dientamoeba & 4.19\% due to Dientamoeba \\
30 & Blastocystis + Giardia & Blastocystis + Giardia+Dientamoeba & \\
33 & Blastocystis & Blastocystis + Dientamoeba \\
61 & Blastocystis & Blastocystis + Dientamoeba \\
72 & Blastocystis & Blastocystis + Dientamoeba & $4.19 \%$ non-concordant \\
120 & Giardia & Giardia+Dientamoeba & \\
35 & Blastocystis + Entamoeba & Blastocystis & \\
6 & Negative & Cryptosporidium \\
48 & Negative & Cryptosporidium \\
49 & Negative & Cryptosporidium \\
80 & Negative & Cryptosporidium \\
119 & Giardia & Negative \\
\hline
\end{tabular}

a Dientamoeba was not included in the laboratory routine diagnostics tests

The limitation of our 5-plex method is the detection of mixed infections with close melting temperatures $(\Delta \mathrm{Tm})$ and one dominant parasite infection. In such a case, only the melting peak of the major parasite would be identified.

However, this limitation is compensated by the possibility to use individual primer sets in single-plex runs to confirm and identify suspected mixed infections. 

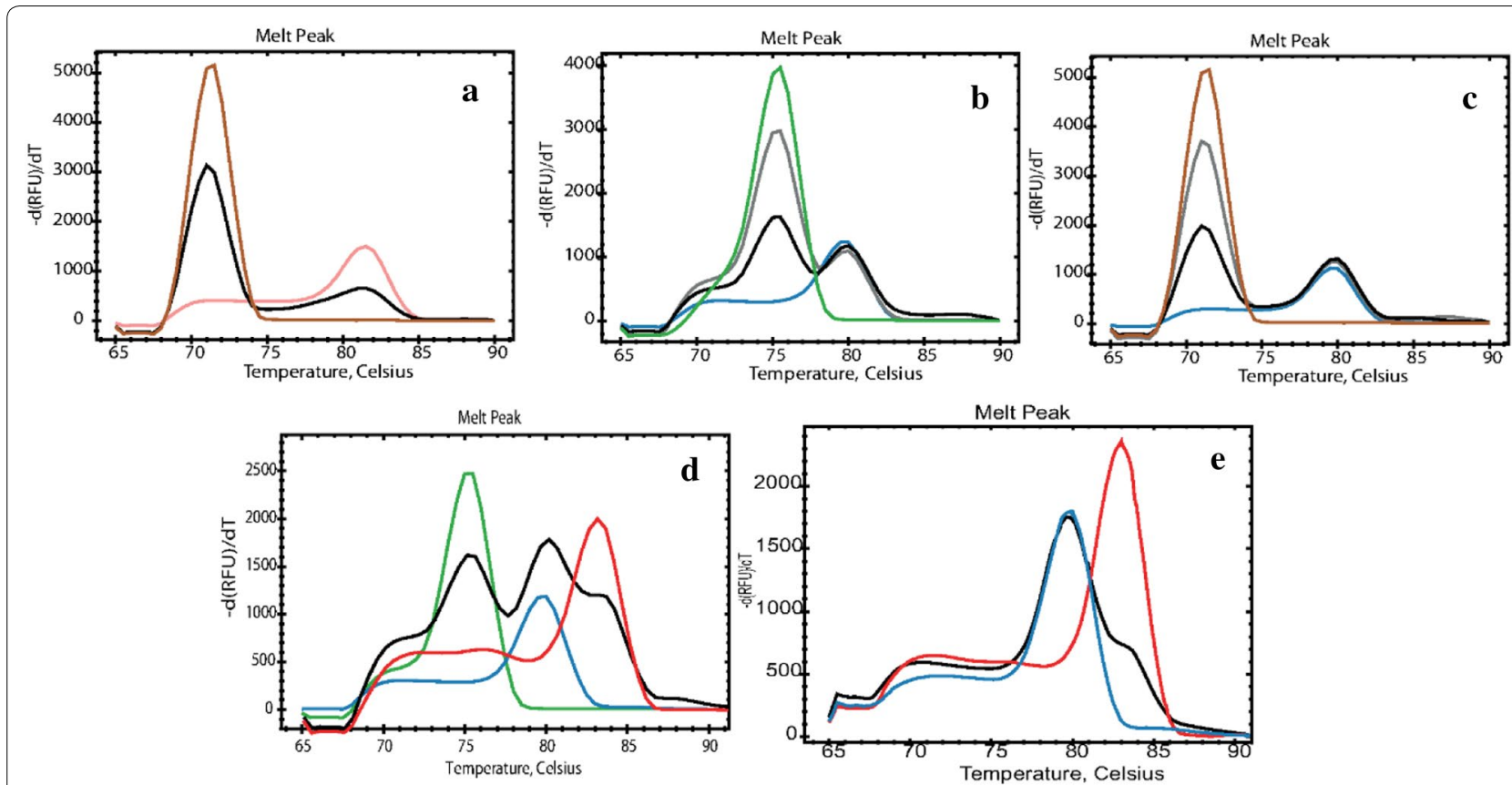

Fig. 4 Melting peaks of mixed infection samples presenting 4 cases of double infection and one case of triple infection. All qPCR runs were done with the 5 primers mix and one color is attributed to each parasite species and the samples (black and grey): Dientamoeba (brown)/Giardia B (pink) a, Entamoeba (green)/Blastocystis (Blue) b, Dientamoeba/Blastocystis c, Entamoeba/Blastocystis/Giardia assemblage A (red) d, and Blastocystis/Giardia assemblage A e. Singleplex runs were done with the target primers to confirm each mixedinfection

\section{Conclusions}

We developed a fast, efficient, cost-effective and easyto-perform 5-plex qPCR-HMR assay for simultaneous detection of Cryptosporidium spp., E. histolytica, both G. intestinalis assemblages (A and B), Blastocystis spp., and $D$. fragilis in stool samples. Moreover, the assay is suited for diagnosing mixed infections and could be expanded to widen its diagnostic potential by the inclusion of further intestinal protozoans (such as Entamoeba coli). It is also adaptable for epidemiological surveys and could contribute significantly to the improvement of patient management and infection control. However, the developed method still requires interlaboratory validation before its implementation in routine diagnostics.

\section{Methods}

\section{Samples and DNA extraction}

The method was evaluated using DNA from 143 stool samples submitted to the Institute of Specific Prophylaxis and Tropical Medicine at the Medical University of Vienna between 2011 and 2018 for diagnostic analysis. The standard diagnostic test of the study samples at our institute included microscopic analysis of stool for all intestinal parasites, immunoassay analysis (Antigens) for G. intestinalis and E. histolytica, and FRET-based
qPCR (DNA) for Blastocystis spp., C. parvum, C. hominis, E. histolytica, E. dispar and G. intestinalis. All samples were anonymized. The Qiagen kit for stool DNA extraction (Qiagen $\mathrm{GmbH}$, Vienna, Austria) was used for the samples' DNA extraction according to manufacturer instructions.

\section{Target selection and primer design}

A fragment of the $18 \mathrm{~S}$ small subunit ribosomal RNA (SSU rRNA) gene (Blastocystis spp., E. histolytica, D. fragilis, Cryptosporidium spp.) and a fragment of the NADP-dependent glutamate dehydrogenase (gdh) gene (G. intestinalis) were selected as targets for the assay due to their frequent use in the literature (Table 1) and were tested with one or two samples of each species (positive samples from the routine diagnostic lab) to confirm their effectiveness. The primers of Cryptosporidium and Blastocystis were selected to cover all species of these parasites as there are many pathogenic species in these genera. As $D$. fragilis was not included in the routine diagnostics at our institute, the long fragment of one of the positive sample (using Verweij et al. primers [9]) was sequenced by Eurofins MWG Synthesis GmbH (Ebersberg, Germany) to confirm the species before designing the short fragment primers. 
New forward and/or reverse primers were designed for each parasite to target a shorter fragment with a melting temperature matching with those of the other parasites for a multiplex qPCR-HRM design (Table 1). All primers were designed using the Primer3 online tool (http://bioin fo.ut.ee/primer3-0.4.0/) and checked for specificity using basic local alignment search. We aligned the sequences with the BioEdit 7.2 Sequence Alignment Editor and produced predictive melting profiles of all fragments using the uMelt BATCH online tool (https://www.dna.utah. edu/umelt/umelt.html) before primers synthesis by Eurofins MWG Synthesis GmbH (Ebersberg, Germany).

\section{PCR and HRM assay}

The long fragments were amplified in the same conditions with $0.5 \mu \mathrm{M}$ primers and the following amplification program: 45 cycles of $95{ }^{\circ} \mathrm{C}$ for $15 \mathrm{~s}, 60{ }^{\circ} \mathrm{C}$ for $30 \mathrm{~s}$ and $68^{\circ} \mathrm{C}$ for $30 \mathrm{~s}$. The PCR products were resolved in $2 \%$ agarose gels and visualized by MIDORI Green Advance (Biozym Scientific GmbH, Hess Oldendorf; Germany) staining.

The multiplex PCR was performed in a volume of $20 \mu$ containing primers, $1 \times$ SsoFast EvaGreen Supermix (BioRad, Hercules, CA) and $4 \mu \mathrm{l}$ DNA $(\leq 20 \mathrm{ng} / \mu \mathrm{l})$. Samples with DNA concentration $>20 \mathrm{ng} / \mu \mathrm{l}$ were diluted 10 times and retested. The optimized primers concentrations were $0.25 \mu \mathrm{M}$ of forward and reverse primers for D. fragilis and E. histolytica, and $0.15 \mu \mathrm{M}$ forward and reverse primers for Cryptosporidium spp., Blastocystis spp., and G. intestinalis.

The PCR was performed on a CFX Connect real-time PCR detection system (BioRad Laboratories, Inc., Singapore) with an initial denaturation step at $95{ }^{\circ} \mathrm{C}$ for $3 \mathrm{~min}$, followed by 40 cycles of $95{ }^{\circ} \mathrm{C}$ for $5 \mathrm{~s}, 57^{\circ} \mathrm{C}$ for $3 \mathrm{~s}$ and $68^{\circ} \mathrm{C}$ for $5 \mathrm{~s}$. The PCR products were subjected to the following melting program: denaturation at $95{ }^{\circ} \mathrm{C}$ for $1 \mathrm{~min}$, cooling to $65^{\circ} \mathrm{C}$ (held for $30 \mathrm{~s}$ ) and continuous heating at $0.5{ }^{\circ} \mathrm{C} / \mathrm{s}$ with fluorescence acquisition from $65{ }^{\circ} \mathrm{C}$ to $90{ }^{\circ} \mathrm{C}$.

\section{HRM plasmids preparation and sequencing}

The short fragments of each parasite were modified and inserted into pET17b through EcoRI/XhoI (Fermentas) restriction sites. The ligated plasmids were used to transform competent XL-1 Blue E. coli competent bacteria. Selected positive clones were cultured, the plasmids purified and sequenced by Eurofins MWG Synthesis GmbH (Ebersberg, Germany). The sequencing data were analyzed using Vector NTI.10 (Invitrogen) software, and the sequences were checked by using the Basic Local Alignment Search Tool (Nucleotide BLAST, https://blast.ncbi. nlm.nih.gov/Blast.cgi) to confirm their identity.

\section{Assay specificity and sensitivity}

We evaluated the method specificity in situ with negative (for all tested protozoans included in this study) stool samples and mixed infections samples, and also with DNA $(\leq 20 \mathrm{ng} / \mu \mathrm{l})$ from the following organisms (using identical PCR conditions): Toxoplasma gondii, Leishmania infantum, Trypanosoma brucei, Trypanosoma cruzi, Babesia divergens, Enterocytozoon bieneusi, Enzephalitozoon cuniculi, Pneumocystis jirovecii, Echinococcus granulosus, Strongyloides stercoralis, Dirofilaria repens, Toxocara canis, Entamoeba dispar, Entamoeba coli, Entamoeba hartmanni, Iodamoeba buetschlii and Ascaris suum. The assay was performed in duplicate with each DNA sample.

The limit of detection (LOD) for each parasite was determined individually under the conditions of the multiplex PCR. The LOD was defined as the measured concentration producing at least $95 \%$ positive replicates [19].

The LOD was assessed by amplifying seven different concentrations $(120,80,40,20,10,5$ and 1 copies/ $\mu$ for Blastocystis spp., Cryptosporidium spp. and D. fragilis; 160, 120, 80, 40, 20, 10 and 5 copies/ $\mu$ for E. histolytica and G. intestinalis) of each plasmid, in twenty (20) replicates on two separate occasions.

The total proportion of positive tests were recorded and subjected to probit regression analysis using $\mathrm{R}$ version 3.4.2 (2017-09-28) via RStudio version Version 1.1.383. Similarly, the boxplots of the melting temperatures (Tm) were also produced using $\mathrm{R}$ via the RStudio version. The Welch's unequal variances t-test was used to compare the difference between arithmetic means of the respective Tm of the amplicons of all five parasite species using $R$.

\section{Abbreviations \\ BLAST: Basic local alignment search tool; DNA: Deoxyribonucleic acid; FRET: Fluorescence resonance energy transfer; HRM: High resolution melting; LOD: Limit of detection; PCR: Polymerase chain reaction; Tm: Melting temperature.}

\section{Acknowledgements \\ Not applicable.}

\section{Authors' contributions}

ALM: Developed the 5-Plex method, analyzed data and wrote the manuscript. RS: Performed laboratory routine diagnostics experiment, discussed data and co-edited manuscript. JW: Discussed data and co-edited manuscript. HA: Provided guidance on parasites selection and co-edited manuscript. UW: Conceived project, discussed data and co-edited manuscript. DL: Conceived project, coordinated cloning experiments, discussed data and co-edited manuscript. All authors read and approved the final manuscript.

\section{Funding}

This work was institutionally supported by the Institute for Specific Prophylaxis and Tropical Medicine, Medical University of Vienna, Austria.

Availability of data and materials

The datasets used and/or analyzed during the current study are available from the corresponding author on reasonable request. 


\section{Ethics approval and consent to participate}

There was no need for obtaining permission for conducting the laboratory experiments for this study. All stool samples were received and handled by our routine diagnostics laboratory for routine diagnostics analysis and DNA extraction. All samples were anonymously used in our multiplex method development and the use of DNA samples is allowed without any ethical approvals from any authorities.

\section{Consent for publication}

Not applicable.

\section{Competing interests}

The authors declare that they have no competing interests.

Received: 2 April 2020 Accepted: 21 May 2020

Published online: 29 May 2020

\section{References}

1. Laude A, Valot S, Desoubeaux G, Argy N, Nourrisson C, Pomares C, et al. Is real-time PCR-based diagnosis similar in performance to routine parasitological examination for the identification of Giardia intestinalis, Cryptosporidium parvum/Cryptosporidium hominis and Entamoeba histolytica from stool samples? Evaluation of a new commercial multiplex PCR assay and literature review. Clin Microbiol Infect. 2016;22:190.e1-8.

2. Norman FF, Comeche B, Chamorro S, Pérez-Molina JA, López-Vélez R. Update on the major imported protozoan infections in travelers and migrants. Future Microbiol. 2020;15(3):213-25.

3. Innes EA, Chalmers RM, Wells B, Pawlowic MC. A one health approach to tackle cryptosporidiosis. Trends Parasitol. 2020;36:290-303.

4. Haque R. Human intestinal parasites. J. Heal Popul Nutr. 2007;25:387-91.

5. Sánchez A, Munoz M, Gómez N, Tabares J, Segura L, Salazar Á, et al. Molecular epidemiology of giardia, Blastocystis and Cryptosporidium among indigenous children from the colombian Amazon basin. Front Microbiol. 2017:8:248.

6. Stensvold CR, van der Giezen M. Associations between gut microbiota and common luminal intestinal parasites. Trends Parasitol. 2018:34:369-77.

7. Garcia LS. Dientamoeba fragilis, one of the neglected intestinal protozoa. J Clin Microbiol. 2016;54:2243-50.

8. Untergasser A, Cutcutache I, Koressaar T, Ye J, Faircloth BC, Remm M, et al. Primer3 Input. Whitehead Inst. Biomed. Res. 2017. http://primer3.ut.ee/.

9. Verweij JJ, Blangé RA, Templeton K, Schinkel J, Brienen EAT, Van Rooyen MAA, et al. Simultaneous detection of Entamoeba histolytica, Giardia lamblia, and Cryptosporidium parvum in fecal samples by using multiplex real-time PCR. J Clin Microbiol. 2004;42:1220-3.

10. Verweij JJ, Rune Stensvold C. Molecular testing for clinical diagnosis and epidemiological investigations of intestinal parasitic infections. Clin Microbiol Rev Am. 2014;27:371-418.

11. Jothikumar N, Da Silva AJ, Moura I, Qvarnstrom Y, Hill VR. Detection and differentiation of Cryptosporidium hominis and Cryptosporidium parvum by dual TaqMan assays. J Med Microbiol. 2008;57:1099-105.
12. Haque R, Roy S, Siddique A, Mondal U, Rahman SMM, Mondal D, et al. Multiplex real-time PCR assay for detection of Entamoeba histolytica, Giardia intestinalis, and Cryptosporidium spp. Am J Trop Med Hyg. 2007;76(4):713-7.

13. Parčina M, Reiter-Owona I, Mockenhaupt FP, Vojvoda V, Gahutu JB, Hoerauf $A$, et al. Highly sensitive and specific detection of Giardia duodenalis, Entamoeba histolytica, and Cryptosporidium spp. in human stool samples by the BD MAX ${ }^{\mathrm{TM}}$ Enteric Parasite Panel. Parasitol Res. 2018;117:447-51.

14. Van Bruijnesteijn Coppenraet LES, Wallinga JA, Ruijs GJHM, Bruins MJ, Verweij JJ. Parasitological diagnosis combining an internally controlled realtime PCR assay for the detection of four protozoa in stool samples with a testing algorithm for microscopy. Clin Microbiol Infect. 2009;15:869-74.

15. Stark D, Al-Qassab SE, Barratt JLN, Stanley K, Roberts T, Marriott D, et al. Evaluation of multiplex tandem real-time PCR for Detection of Cryptosporidium spp, Dientamoeba fragilis, Entamoeba histolytica, and Giardia intestinalis in clinical stool samples. J Clin Microbiol. 2011;49:257-62.

16. Eischeid AC. SYTO dyes and EvaGreen outperform SYBR Green in realtime PCR. BMC Res Notes. 2011:4:263.

17. Bustin SA, Benes V, Garson JA, Hellemans J, Huggett J, Kubista M, et al. The MIQE guidelines: minimum information for publication of quantitative real-time PCR experiments. Clin Chem. 2009;55:611-22.

18. Marangi M, Giangaspero A, Lacasella V, Lonigro A, Gasser RB. Multiplex PCR for the detection and quantification of zoonotic taxa of Giardia, Cryptosporidium and Toxoplasma in wastewater and mussels. Mol Cell Probes. 2015:29:122-5.

19. Forootan A, Sjöback R, Björkman J, Sjögreen B, Linz L, Kubista M. Methods to determine limit of detection and limit of quantification in quantitative real-time PCR (qPCR). Biomol Detect Quantif. Elsevier GmbH. 2017;12:1-6.

20. Hamzah Z, Petmitr S, Mungthin M, Leelayoova S, ChavalitshewinkoonPetmitr P. Differential detection of Entamoeba histolytica, Entamoeba dispar, and Entamoeba moshkovskii by a single-round PCR assay. J Clin Microbiol. 2006;44:3196-200.

21. Lalonde LF, Gajadhar AA. Detection and differentiation of coccidian oocysts by real-time PCR and melting curve analysis. J Parasitol. 2011:97:725-30.

22. Poirier P, Wawrzyniak I, Albert A, El Alaoui H, Delbac F, Livrelli V. Development and evaluation of a real-time PCR assay for detection and quantification of Blastocystis parasites in human stool samples: prospective study of patients with hematological malignancies. J Clin Microbiol. 2011:49:975-83.

23. Read CM, Monis PT, Thompson RCA. Discrimination of all genotypes of Giardia duodenalis at the glutamate dehydrogenase locus using PCRRFLP. Infect Genet Evol. 2004;4:125-30.

\section{Publisher's Note}

Springer Nature remains neutral with regard to jurisdictional claims in published maps and institutional affiliations.

Ready to submit your research? Choose BMC and benefit from

- fast, convenient online submission

- thorough peer review by experienced researchers in your field

- rapid publication on acceptance

- support for research data, including large and complex data types

- gold Open Access which fosters wider collaboration and increased citations

- maximum visibility for your research: over 100M website views per year

At BMC, research is always in progress.

Learn more biomedcentral.com/submissions 